\title{
Antineutrophil cytoplasmic antibodies and their relationship with disease activity and presence of staphylococcal superantigens in nasal swabs in patients having granulomatosis with polyangiitis: results of a study involving 115 patients from a single center
}

\author{
Justyna Fijolek $^{1}$ (D) E. Wiatr ${ }^{1}$ - V. Petroniec ${ }^{2} \cdot$ E. Augustynowicz-Kopec $^{2} \cdot$ M. Bednarek ${ }^{3} \cdot$ D. Gawryluk $^{1} \cdot$ \\ K. Roszkowski-Sliz ${ }^{1}$
}

Received: 30 March 2019 / Revised: 5 June 2019 / Accepted: 12 July 2019/Published online: 23 July 2019

(C) The Author(s) 2019

\begin{abstract}
Objective Antineutrophil cytoplasmic antibodies (ANCAs) are considered a risk factor for granulomatosis with polyangiitis (GPA) exacerbation, especially when staphylococcal superantigens (SAgs) are present in nasal swabs. Their role in monitoring disease activity remains controversial. This study determined the relationship of ANCAs with disease activity and presence of SAgs in GPA patients. Methods Among a total of 115 GPA patients hospitalized in the period 2009-2016, we investigated the presence of SAgs and ANCA concentration. Blood samples and nasal swabs were taken at each visit (referred further to as episodes). Disease activity was assessed using the Birmingham Vasculitis Activity Score (BVAS).

Results We analyzed 362 episodes. ANCAs were detected in 215 (59.4\%), while SAgs were detected in 126 (34.8\%) episodes. We found a significant correlation between the presence of ANCAs and disease activity $(p=0.0032)$, as well as between their level and GPA severity $(r=0.25363, p=0.000001)$. We also determined that an ANCA values $\geq 138 \mathrm{Ru} / \mathrm{ml}$ were an indicator of active disease with high specificity and low sensitivity (84.4\% and 37.3\%, respectively). The relationship between ANCA presence and the presence of SAgs was not confirmed; however, when SAgs were analyzed based on the different types, ANCA levels were found to be significantly higher in the group with SAg type B $(p=0.031)$.

Conclusions There was no detectable evidence for the association between ANCA level and the presence of SAgs. Although monitoring ANCA levels as a marker of disease activity may be clinically relevant, GPA management cannot proceed on the basis of ANCA levels alone.

\section{Key Points}

- ANCA concentration usually correlates with GPA activity, although in half of patients, ANCAs persist despite effective treatment and clinical remission.

- ANCA values of $138 \mathrm{Ru} / \mathrm{ml}$ seem to be an indicator of active disease with high specificity, but low sensitivity.

- Although there is a relevance for ANCA monitoring as a marker of disease activity, GPA management cannot be based on ANCA levels alone.

- The suspected clinical correlation between ANCA formation and SAg presence in nasal swabs is not obvious and requires further investigations.
\end{abstract}

Keywords Antineutrophil cytoplasmic antibodies · Disease activity · Granulomatosis with polyangiitis · Staphylococcal superantigens

Justyna Fijolek

jfijolek@op.pl

1 The Third Department of Pneumonology, National Tuberculosis and Lung Diseases Research Institute, Plocka St 26,

01-138 Warsaw, Poland

2 Department of Microbiology, National Tuberculosis and Lung Diseases Research Institute, Warsaw, Poland

3 The Second Department of Pneumonology, National Tuberculosis and Lung Diseases Research Institute, Warsaw, Poland

\section{Introduction}

Antineutrophil cytoplasmic antibodies (ANCAs) are a group of autoantibodies directed against proteins predominantly expressed in the cytoplasmic granules of neutrophils. They were first reported in serum from a few patients with segmental necrotizing glomerulonephritis in 1982 [1]. The data were largely disregarded until Van der Woude et al. [2] described similar patterns of autoantibodies in patients with systemic vasculitis, including granulomatosis with polyangiitis 
(GPA) - formerly called Wegener's granulomatosis - in 1985. The disease is characterized by granulomatous inflammation of the respiratory tract, necrotizing small-vessel vasculitis, and glomerulonephritis; ANCAs are directed predominantly against the myeloid proteinase 3 (PR3) and myeloperoxidase (MPO) is frequently observed as a hallmark of this condition [3].

Previous studies have demonstrated that ANCAs cause neutrophil activation leading to vascular endothelial injury to small blood vessels [4]. Antigens, such as microbial components, stimulate ANCA autoantigen expression on the membrane surface of neutrophils. ANCAs are developed and released from B cells; they can bind to the ANCA autoantigens and activate neutrophils. Once activated, neutrophils attach to the endothelium of the blood vessels and release reactive oxygen species, inflammatory cytokines, toxic substances, and neutrophil extracellular traps (NETs), leading to systemic vasculitis and injuries to multiple organs $[4,5]$.

It has been observed that $60-70 \%$ of patients with GPA are chronic nasal Staphylococcus aureus (S. aureus) carriers, in contrast to the $20-30 \%$ of healthy individuals. Therefore, S. aureus and its superantigens (SAgs) have been indicated to play a special role in the pathogenesis of GPA, leading to ANCA formation [6-9]. Pendergraft et al. [10] presented evidence that complementary PR3, which shows homology with certain $S$. aureus-derived peptides, may induce antibodies to PR3, leading to the development of ANCA and activation of GPA by molecular mimicry.

Although the diagnostic importance of ANCAs in cases involving GPA has been widely recognized $[11,12]$, their role in monitoring disease activity remains controversial. Some studies have reported that ANCA levels correlate with disease activity [13]. However, other studies have demonstrated no relationship between ANCA levels and disease activity in patients with GPA [14]. According to some findings, persistence of ANCAs and presence of SAgs are associated with disease activity and probably are interrelated [9].

In a previous study [15], we analyzed the relationship between the presence of SAgs in nasal swabs and GPA activity, but the aim of the current study was to determine the relationship between ANCA concentration and disease activity, while taking into account the association between ANCA formation and the presence of SAgs in nasal swabs in patients with GPA.

\section{Materials and methods}

The retrospective study was conducted in compliance with the principles of the Declaration of Helsinki. Ethics Committee approval was not obtained in accordance with Polish law, which states that formal Ethics Committee approval is not required in the context of standard care. Informed consent of the participants was waived.
The study cohort consisted of 115 patients with GPA having positive nasal swabs, who were hospitalized in the National Tuberculosis and Lung Diseases Research Institute during the period of 2009-2016. GPA was diagnosed on the basis of clinical features consistent with vasculitis and/or biopsy results consistent with vasculitis and/or a positive ANCA test in accordance with the Chapel Hill Consensus Conference 2012 nomenclature [16]. Disease activity was assessed using the Birmingham Vasculitis Activity Score (BVAS) [17] during each visit, referred further to as episodes. Non-active disease was defined as the absence of symptoms or signs attributable to active vasculitis (BVAS $=0$ ), in combination with a normal serum $\mathrm{C}$-reactive protein (CRP) concentration. This group included patients who had undergone immunosuppressive treatment and those who were under immunosuppression, in whom disease remission had been obtained. Active disease was recognized in newly diagnosed patients, and in patients having relapse or in those in whom progression of the disease was observed (according to BVAS, clinical and radiological syndromes, and laboratory findings). Cases involving minor lung radiological progression without clinical syndromes were also identified as having an active disease. In patients with GPA limited to subglottic stenosis (SGS), the disease activity indicator was dyspnea - requiring surgical intervention - the intratracheal dilation-injection technique during "rigid" bronchoscopy. Treatment included the administration of corticosteroids (CSs) and cyclophosphamide (CYC), and then administration of azathioprine (AZA) or methotrexate (MTX) - for the maintenance of remission. The treatment regimen was maintained for 18-24 months. Patients with severe and recurrent disease received rituximab, but patients with GPA limited to SGS were mainly treated locally [18]. Some patients received trimetophrim/sulfamethoxazole (T/S) in prophylactic doses (960 mg three times a week), while others additionally received local treatment using mupirocin.

\section{ANCA detection}

All patients included in this study were examined for ANCA. Sera were collected during each visit. First, serial serum dilutions (starting at $1 / 20$ ) were tested by indirect immunofluorescence (IIF) on ethanol-fixed human neutrophils, and then PR3ANCA and MPO-ANCA levels were assessed using a standard commercial enzyme-linked immunosorbent assay (ELISA) kit. The assays were calibrated in relative units per milliliter $(\mathrm{Ru} /$ $\mathrm{ml}$ ). Values $>20 \mathrm{Ru} / \mathrm{ml}$ were interpreted as positive results, while those $\leq 20 \mathrm{Ru} / \mathrm{ml}$ were interpreted as negative results.

\section{Nasal cultures and SAg typing}

Nasal swabs were taken during each visit (episode). The method of $S$. aureus isolation and the assessment of eneterotoxicity have been described in detail in a previous study [16]. 


\section{Statistical analyses}

Statistical analyses were performed using Statistica version 10 software (StatSoft, Inc., USA). Differences were considered significant when $p<0.05$ was obtained. Data distribution was analyzed using the Kologomorov-Smirnov test with Lilliefors correction. Homogeneity of variance was assessed using Levene's test. Quantitative data were described using mean $\pm \mathrm{SD}$. Intergroup characteristics were described using a $t$ test or analysis of variance (ANOVA) and $F$ tests, if a variable demonstrated normal distribution and homogenous variance. Otherwise, the Mann-Whitney $U$ test or Kruskall-Wallis ANOVA was applied.

Categorized, qualitative, between-group analyses were performed using Pearson's chi-square $\left(\chi^{2}\right)$ test with appropriate corrections for $\mathrm{N}$.

A receiver operating characteristic (ROC) curve analysis was applied for predictive analyses of qualitative data. The Medical Package version 2.0 (StatSoft, Inc., USA, 2011) for Statistica was used. The area under the ROC curve, likelihood ratio (LR), sensitivity, and specificity were calculated. For cutoff value, the parameter with the highest LR was chosen.

\section{Results}

Among the 115 patients with GPA and coexisting S. aureus in nasal swabs, there were 55 women $(47.8 \%)$ and 60 men $(52.2 \%)$, with a mean age during the first episode of $45.0 \pm$ 17.0 and $44.5 \pm 13.6$ years, respectively. There were no statistically significant difference depending on the sex $(p=$ $0.85)$. In 98 patients $(85.2 \%)$, the disease was generalized, while in 17 patients, the disease was limited to SGS (14.8\%). In total, 362 episodes were analyzed in the study. Active disease was found in $32.6 \%$ (118) of episodescompared with $67.4 \%$ (244) episodes of non-active disease. There were no significant differences in terms of patient age $(45.8 \pm 15.1$ and $45.8 \pm 16.1, p=0.28)$ and $\operatorname{sex}(p=0.195)$. The characteristics of the study patients are shown in Table 1.
Table 1 Clinical characteristics of the 115 study patients ( 362 episodes)

\begin{tabular}{lc}
\hline Patients' characteristics & No. $(\%)$ \\
\hline Male patients & $60(52.2 \%)$ \\
Female patients & $55(47.8 \%)$ \\
Male episodes & $168(46.4 \%)$ \\
Female episodes & $194(53.5 \%)$ \\
Generalized disease patients & $98(85.2 \%)$ \\
Limited disease-SGS patients & $17(14.8 \%)$ \\
Generalized disease active episodes & $96(81.4 \%)$ \\
Generalized disease non-active episodes & $217(88.9 \%)$ \\
Limited disease active episodes & $22(18.6 \%)$ \\
Limited disease non-active episodes & $27(11 \%)$ \\
All active episodes & $118(32.6 \%)$ \\
All non-active episodes & $244(67.4 \%)$ \\
BVAS & \\
0 & $244(67.4 \%)$ \\
1-3 & $61(16.9 \%)$ \\
4-6 & $41(11.3 \%)$ \\
$7-10$ & $16(4.4 \%)$ \\
S. aureus-positive nasal swab patients & $115(100 \%)$ \\
SAg (+) episodes & $126(34.8 \%)$ \\
SAg (-) episodes & $236(65.2 \%)$ \\
Only SAg SEA-positive nasal swab patients & $15(13 \%)$ \\
Only SAg SEB-positive nasal swab patients & $2(1.7 \%)$ \\
Only SAg SEC-positive nasal swab patients & $12(10.4 \%)$ \\
Only SAg SED-positive nasal swab patients & $3(2.6 \%)$ \\
Only SAg TSST-1-positive nasal swab patients & $6(5.2 \%)$ \\
More than one SAg positive in a single nasal swab patients & $18(15.6 \%)$ \\
ANCA (+) episodes & $215(59.4 \%)$ \\
ANCA (-) episodes & $147(40.6 \%)$ \\
T/S treatment patients & $45(39 \%)$ \\
Yes & $70(60.9 \%)$ \\
No & $123(34 \%)$ \\
T/S treatment episodes & $239(66 \%)$ \\
Yes & $115(100 \%)$ \\
No & $362(100 \%)$ \\
All patients & \\
All episodes (visits) & \\
\hline &
\end{tabular}

SGS, subglottic stenosis; BVAS, Birmingham Vasculitis Activity Score; S. aureus, Staphylococcus aureus; SAg, staphylococcal superantigens; $S E A$, staphylococcal enterotoxicity type A; $S E B$, staphylococcal enterotoxicity type B; SEC, staphylococcal enterotoxicity type C; SED, staphylococcal enterotoxicity type D; TSST-1, toxic-shock syndrome toxin 1; ANCA, antineutrophil cytoplasmic antibodies; $T / S$, trimetophrim/sulfamethoxazole 
ANCAs were found in 215/362 (59.4\%) episodes, 83 (70.3\%) episodes of active disease and in $132(54.1 \%)$ of non-active disease. Predominantly, the episodes involved PR3-ANCA, while MPO-ANCA was detected only in seven episodes (three patients). In 35 (29.7\%) episodes of active disease and in $112(45.9 \%)$ episodes of non-active disease, ANCAs were not detected. Figure 1 shows a detailed distribution of ANCA concentration depending on the BVAS assessed during each episode. The statistical analysis confirmed a significant correlation between the presence of ANCAs and active GPA $(p=0.0032)$, as well as between their concentration and disease severity ( $R=0.25363, p=0.000001)$ (Fig. 2). Additionally, the ROC analysis (Fig. 3) demonstrated that for a cut-off ANCA level of $138 \mathrm{Ru} / \mathrm{m}$, the optimal LR obtained for distinguishing between active and non-active disease was 2.39 , with a sensitivity of $37.3 \%$ and a specificity of $84.4 \%$. Interestingly, a significant correlation between ANCA concentration and patient sex was detected (men $>$ female, $p=0.035$ ). When we consider the ANCA presence in particular patients, among the all 115 patients, in 29 (25.2\%), ANCAs were not detected during the 7-year follow-up period, and in 29 (25.2\%), ANCA status was variable (for example, they were detected in active disease and become negative after treatment). In 57 patients (49.6\%), ANCAs were found during each visit (episode). Regarding to 362 episodes analyzed in the study, in 147 (40.6\%), ANCAs were negative.

The presence of at least one type of SAg was found in 126 samples (34.8\%) from 56 patients (48.7\%); among the 17 patients with GPA limited to SGS, SAgs were detected in 6 (35.3\%). In all samples, the most commonly found SAg was SEA (73/126 episodes, 60\%), but the rarest was SEB (eight among the 126 episodes, $6.3 \%$ ). We found no significant correlation between the presence of SAgs in nasal swabs and
ANCA concentration, but when SAgs were analyzed based on the different types, the ANCA level was significantly higher in the group with SEB expression $(p=0.031)$ (Table 2). Interestingly, the average ANCA concentration increased with the number of SAg types detected in the single sample, but the relation was not statistically significant (Fig. 4).

\section{Discussion}

Results of our study confirmed the significant correlation between the presence of ANCA and disease activity $(p=$ 0.0032). ANCAs have been recognized as important biomarkers to aid in the diagnosis of patients with GPA [19]. Therefore, patients presenting with clinical features of systemic small-vessel vasculitis, with suspected GPA, are routinely tested for ANCA. Increasing clinical and experimental evidence supports the pathogenic role of ANCA in GPA, mainly through ANCA-induced activation of primed neutrophils and monocytes, leading to destructive vascular necrosis [5]. Therefore, as expected, high circulating titers of ANCA should be typically seen during active disease, with subsequent falls during therapy. On the other hand, some findings suggested that in cases with only 50\% ANCA elevation, relapse was observed, and around half of the relapses occured even in the absence elevations in the ANCA titer [20].

Thus, the correlation between ANCA presence and disease activity is not evident and still remains controversial. In a study of 100 patients with GPA and PR3-ANCAs, Boomsma et al. [21] observed that 26 of the 33 relapses that occurred during the study period were preceded by a rise in PR3-ANCA levels. Next, Stegeman et al. [22] showed that the persistence of ANCA confers a relative risk of 9.0 for relapse
Fig. 1 The detailed distribution of ANCA concentration in GPA study patients (362 episodes) depending on the disease activity assessed using the BVAS

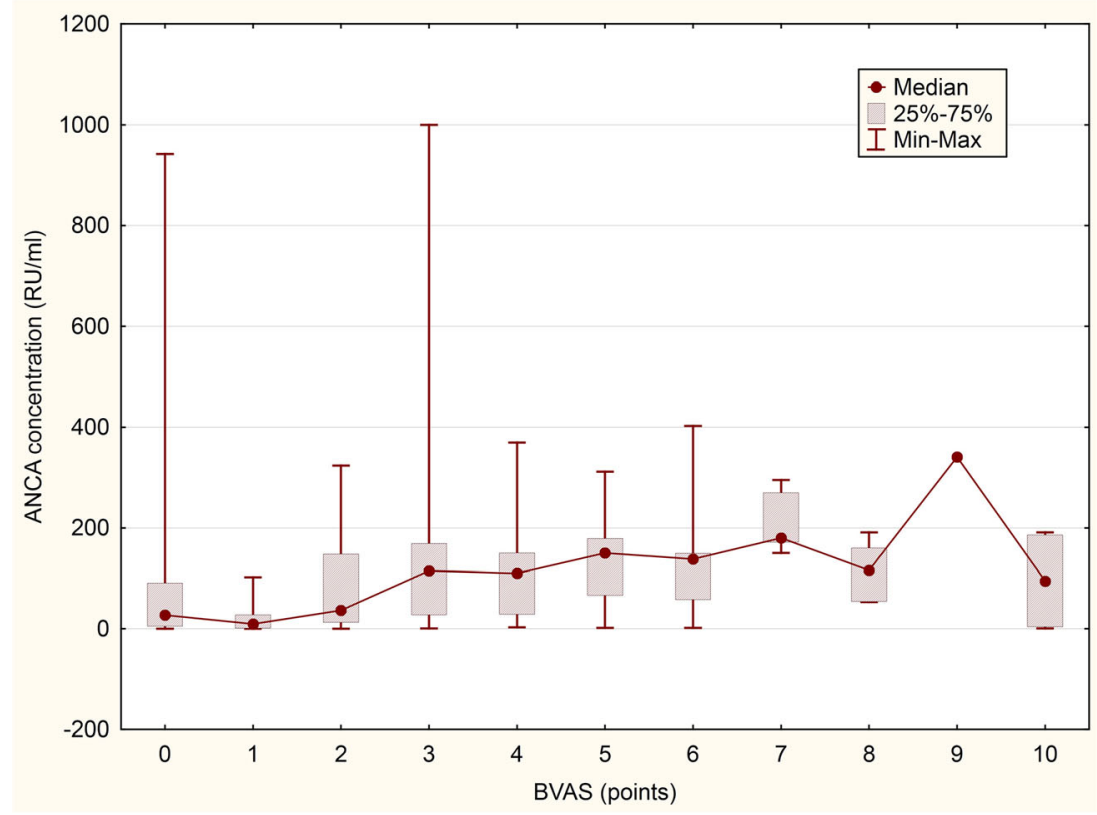


Fig. 2 The correlation between ANCA concentration and GPA activity in study patients (362 episodes)

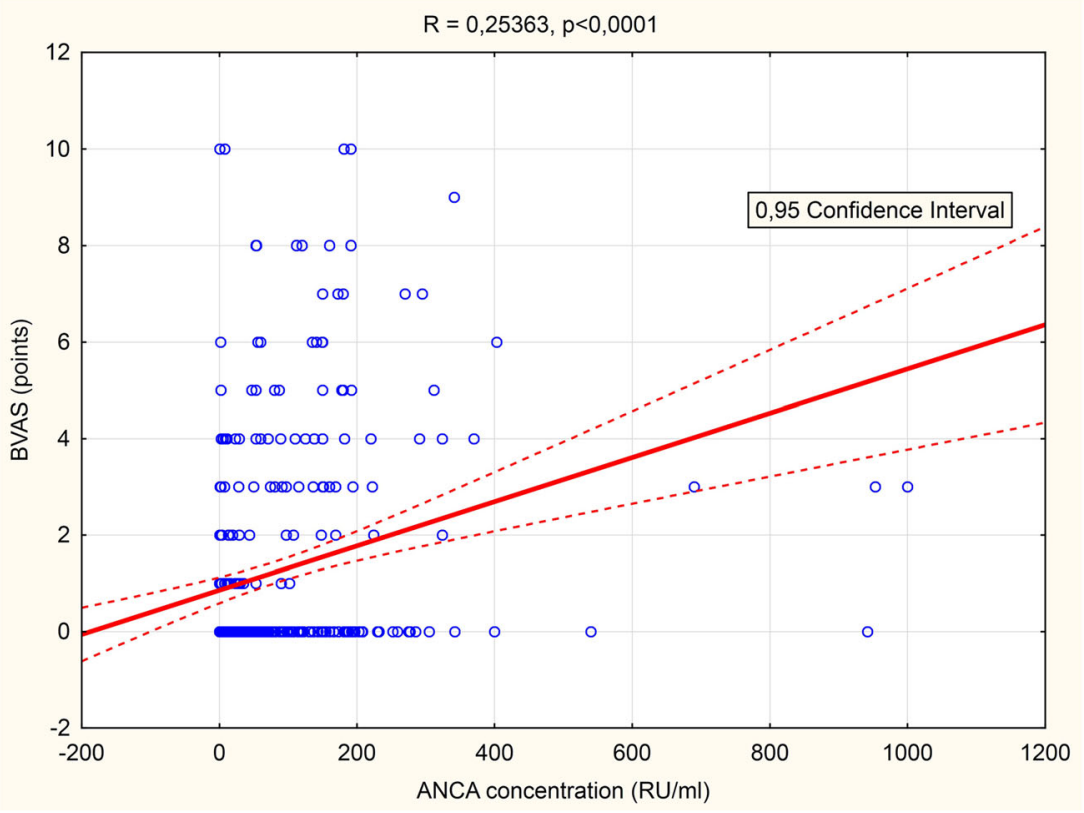

and disease progression. Similar results were reported by Han et al. [23] in their retrospective study. Authors have demonstrated that serial measurement of ANCA titers in patients with GPA during remission can help predict relapses. Moreover, pre-emptive increase in immunosuppression, following a fourfold titer rise, reduced the risk of relapses. On the other hand, there are findings showing that changes in serial PR3-ANCA titers not always correlated with changes in disease activity. A meta-analysis of nine studies concluded that neither the increase nor the persistence of ANCA in patients

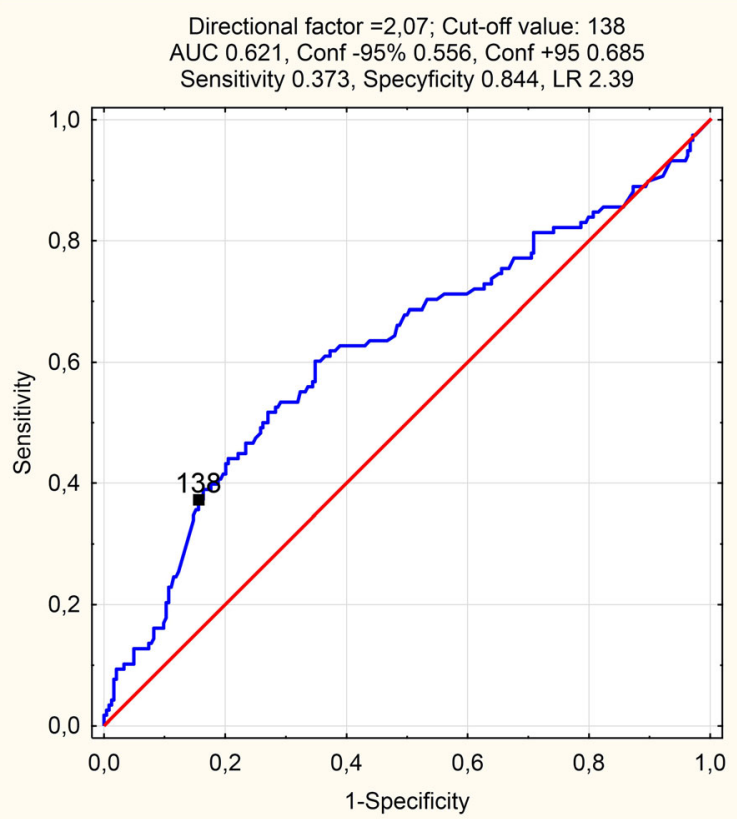

Fig. 3 ROC plot for ANCA concentration, an effect on GPA activity in study patients with GPA who achieved remission is highly predictive of relapse or disease progression [24]. In turn, in the restrospective study by Thai et al. [25], individual ANCA-profile analysis revealed that only for $60 \%$ of GPA patients, clinical outcomes and ANCA-titer changes were closely associated, that is, ANCAs were always present during active disease and absent during remission. On the other hand, Girard et al. [26] reported that in $79 \%$ of the patients with GPA included in the prospective study, the clinical course improved with the disappearance of ANCAs and deteriorated with their persistence or increased titer. Some authors concluded that although GPA progression was usually more frequent when ANCA remained positive or relapsed, discordance between ANCA levels and disease activity was not unusual. Fussner et al. [27] confirmed the findings of earlier publications, which have shown that an increase in PR3-ANCA levels is not a very sensitive and specific predictor of a subsequent worsening of GPA. However, in their study, an increase in PR3-ANCA titers was associated with severe relapses, particularly in patients with renal involvement and alveolar hemorrhage.

In our study, the frequency of ANCA detection was significantly higher in the group of active disease episodes than in the group with non-active ones ( $70.3 \%$ vs $54.1 \%)$; moreover, the ANCA concentration was significantly higher in more severe GPA $(R=0.25363 ; p=0.000001)$. These results are consistent with those of other publications. Sinico et al. [28] analyzing disease status and its correlation with ANCA levels in 71 patients with ANCA-associated vasculitis (AAV) showed that changes in ANCA titers were strongly associated with changes in the BVAS, mainly in PR3-ANCA patients. Similarly, Houben et al. [29] demonstrated that higher ANCA levels in GPA patients are associated with multiple organs and 
Table 2 The impact of the SAg on the ANCA level in a total of 115 GPA patients (362 episodes)

\begin{tabular}{lllllll}
\hline ANCA level mean $\pm \mathrm{SD}$ & SAg + & SEA + & SEB + & SEC+ & SED+ \\
\hline Yes & $99.9 \pm 150.7$ & $106.9 \pm 177.3$ & $240.5 \pm 300.8$ & $69.3 \pm 75.6$ & $80.1 \pm 80.6$ & $114.7 \pm 174.9$ \\
No & $71.23 \pm 103.6$ & $74.7 \pm 103.8$ & $77.1 \pm 112.7$ & $82.2 \pm 125.8$ & $81.2 \pm 123.4$ & $77.5 \pm 115.2$ \\
$p$ & $0.074(\mathrm{~ns})$ & $0.23(\mathrm{~ns})$ & 0.031 & $0.098(\mathrm{~ns})$ & $0.99(\mathrm{~ns})$ & $0.23(\mathrm{~ns})$ \\
\hline
\end{tabular}

$A N C A$, antineutrophil cytoplasmic antibodies; $S A g$, staphylococcal superantigen; $S E A$, staphylococcal enterotoxin type A; $S E B$, staphylococcal enterotoxin type B; $S E C$, staphyloccal enterotoxin type C; $S E D$, staphylococcal enterotoxin type D; TSST-1, toxic-shock syndrome toxin $1 ; n s$, not significant

systemic involvement. Interestingly, in our material, ANCA values $\geq 138 \mathrm{Ru} / \mathrm{ml}$ were revealed as an indicator for patients having active disease (LR of 2.39), with a high specificity, but a low sensitivity ( $84.4 \%$ and $37.3 \%$, respectively), which could be affected by the type of laboratory assay being used as well as by the presence of seronegative ANCA disease in study cohort. So far, due to the lack of standarization of ANCA binding units, there are no criteria (such as ANCA binding unit or change in level) that define clinical remission or disease worsening, and cut-off levels for remission or disease worsening are unlikely because of different testing methodologies, epitopes or antigen preparations, and binding conditions. Therefore, so far, no assay currently suggests when treatment can be reduced or ceased, or when a relapse or disease worsening occurs. It seems, that our study is the first to indicate the critical level of ANCA, which is necessary to determine active and non-active GPA.

On the other hand, ANCAs were detected in 54.1\% (132/ 244) of non-active GPA episodes, and they were absent in almost $30 \%(35 / 118)$ of active disease episodes analyzed in the present study. These findings indicate that ANCAs, although involved in the pathogenesis of GPA, not always correlate with disease activity. Some studies strongly supported this conclusion. Finkielman et al. [30] in their prospective, observational study involving 156 patients with GPA demonstrated that changes in ANCA levels explained less than $10 \%$ of the variation in disease activity. Regarding these results, Verstockt et al. [31] concluded that ANCA monitoring in patients with GPA has limited value and targeted determination of ANCA levels may be useful, only, if a disease worsening is clinically suspected. These results suggest the possibility that the presence of ANCA in AAV represents an epiphenomenon, accompanying but not necessarily driving the disease. This is because ANCAs are not detectable in approximately $20 \%$ of GPA cases [32], and clinical benefit following B celldepleting rituximab therapy is often seen before a significant reduction in ANCA titer [33] and has also been reported in ANCA-negative cases [34].

Another aspect of this study concerned the clinical relation between ANCA formation and SAg presence in nasal swabs in patients with GPA. There is considerable evidence that ANCAs are involved in the pathogenesis of AAV, but the mechanism leading to their formation is unclear. Both experimental [35] and epidemiological [6] studies have provided strong supportive evidence to the concept that infections may trigger exacerbations of ANCA-mediated disease and
Fig. 4 An average ANCA concentration depending on the number of the SAg presence in a single nasal swab in study patients (362 episodes)

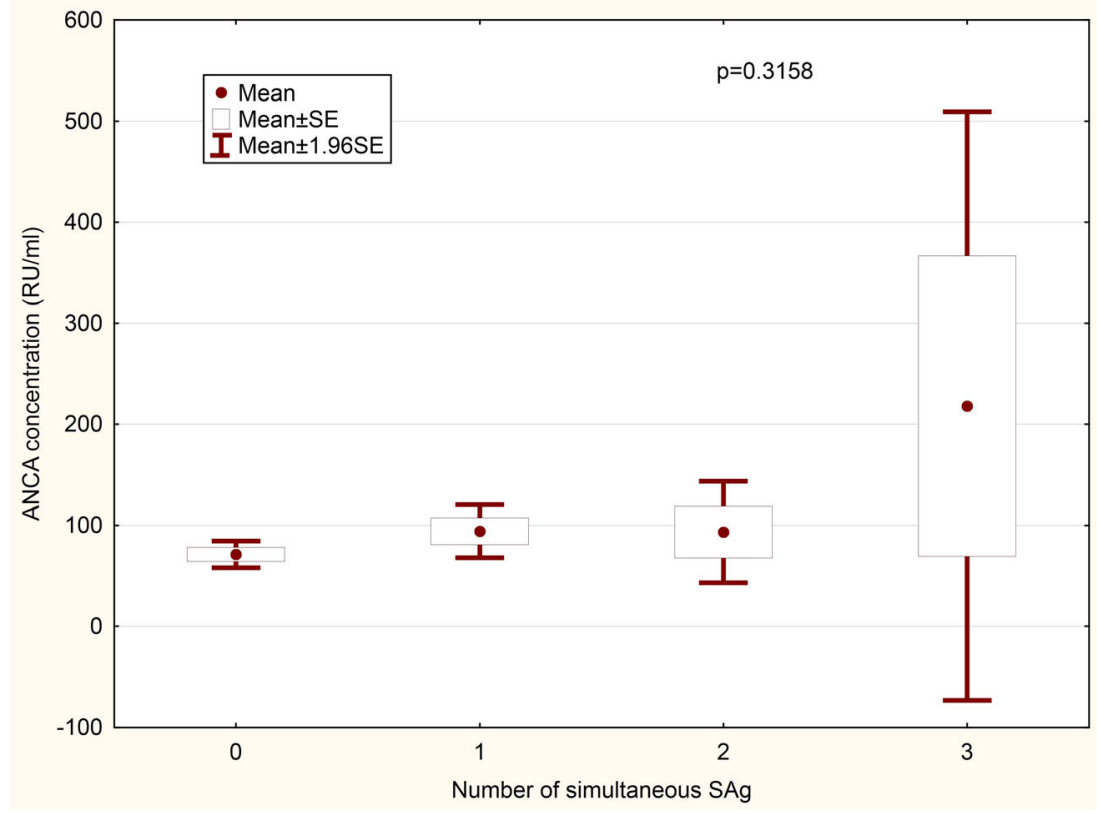


may even be the main pathogenetic mechanism underlying ANCA formation. One factor that has been linked to GPA and the presence of anti-PR3 antibodies in particular is nasal carriage of $S$. aureus $[5,36]$, especially its SAgs, because their role as extremely potent immunostimulatory molecules $[9$, 37]. The mechanisms underlying the relationship between S. aureus and ANCA formation remain unclear, but numerous theories have been postulated, such as autoantigen complementarity or molecular mimicry with production of ANCAs directed against the human lysosome membrane protein hLAMP-2 [8, 38]. Recently, multiple sources of evidence have reinforced the concept of the central pathogenetic role of NETs in the pathogenesis of AAV as a source for the formation of ANCA [39].

There were several clinical studies evaluating a critical role of SAgs for GPA activity [15, 40, 41], but none, so far, investigated, whether there is a relation between SAgs presence and ANCA levels in patients with GPA. Our study seems to be the first analyzing this association. The results did not confirm a suspected correlation between ANCA levels and presence of SAg, although ANCA average concentration increased with the number of SAgs in the single sample. Interestingly, when SAgs were analyzed based of the different types, ANCA levels were significantly higher in the group with SEB expres$\operatorname{sion}(p=0.031)$. The results should be regarded very carefully. First, the number of SEB (+) episodes was small (eight among the 362 episodes, $6.3 \%$ ). Second, there are preliminary results and undoubtedly require further investigations in prospective studies. However, our findings support that the exact mechanism of ANCA formation is not clear and indicates that $S$. aureus may play only a secondary pathogenic role in GPA.

It has been recently reported that two types of ANCAs: PR3 and MPO, may identify distinct patient categories within AAV, associated with a particular genetic background, immune pathology, clinical presentation, and response to treatment and prognosis [42-44]. MPO-ANCA vasculitis is associated with a higher risk of more severe clinical involvements, like renal disease and alveolar hemorrhage, while PR3-ANCA is associated with higher risk of upper airway involvement (ear, nose, throat-ENT) and ocular involvement. Patients with MPOANCA have relapse less frequently than those with PR3ANCA, and might benefit in the future from shorter-term drug administration with less ANCA monitoring, while PR3ANCA-positive patients are clearly at risk of relapse [43, 44]. In our study, patients predominantly involved PR3-ANCA, while MPO-ANCA was detected only in three among the 115 GPA patients (seven among the 362 episodes, $2 \%$ ); therefore, a comparative analysis with the group with PR3-ANCA positivity was not possible. However, two of the patients had GPA limited to SGS, but characterized by a different course. The first initially had severe and recurrent SGS, which ended in the development of multiorgan GPA, while the second still had limited and mild disease. In the third patient, GPA was limited to pulmonary manifestation with minor progression in radiological studies, without clinical symptoms of vasculitis. Surprisingly, not only patients with PR3-ANCA-positive GPA, but also MPO-AAV, carried $S$. aureus isolates that are prevalent in the general population. Interestingly, MPO-ANCAassociated $S$. aureus isolates seem to be genetically distinct from healthy control- and PR3-ANCA-associated isolates [45]. In our study, SAgs in nasal swabs were found in two of the MPO-ANCA GPA patients (four episodes) and only in one of them, the presence of SAgs correlated with ANCA positivity and disease activity. In five among the seven MPO-ANCA episodes, ANCA relapsed, when GPA symptoms worsened.

This study has several limitations such as its retrospective design and the absence of systemic examinations of ANCA concentrations. In addition, ANCA specificity was assessed with a routine ELISA kit employing a mixture of human native and human recombinant PR3. However, all blood samples were assessed in one certified laboratory; therefore, the ANCA results are unified and comparable. Moreover, some patients received chronic antimicrobial treatment with $\mathrm{T} / \mathrm{S}$, which could have contributed to reduced staphylococcal enterotoxicity. Finally, most of the patients were PR3ANCA positive; therefore, comparison of results with MPO was not possible.

In conclusion, our results confirmed the correlation between ANCA presence and GPA activity: higher ANCA levels are associated with more severe GPA. To the best of our knowledge, this is the first study to define the critical level of ANCAs, which in turn will help determine active and nonactive GPA. Our study shows that ANCA values of $138 \mathrm{Ru} / \mathrm{ml}$ are an indicator of active disease with high specificity, but low sensitivity. In $54.1 \%$ patients, ANCAs persist despite effective treatment and clinical remission of disease; therefore, serial ANCA titers for monitoring disease activity have limited value and remain controversial. To our knowledge, this is also the first study to estimate the clinical association between ANCA formation and SAg presence in nasal swabs in GPA patients. The results did not confirm the suspected correlation, although ANCA average concentration increased with the number of SAgs in a single sample. Furthermore, when individual types of SAgs were analyzed separately, ANCA levels were significantly higher in the group with SEB positivity, but because of the small number of this kind of episodes, the results should be analyzed carefully and require further investigations.

Compliance with ethical standards The retrospective study was conducted in compliance with the principles of the Declaration of Helsinki. Ethics Committee approval was not obtained in accordance with Polish law, which states that formal Ethics Committee approval is not required in the context of standard care. Informed consent of the participants was waived.

Disclosures None. 
Open Access This article is distributed under the terms of the Creative Commons Attribution 4.0 International License (http:// creativecommons.org/licenses/by/4.0/), which permits unrestricted use, distribution, and reproduction in any medium, provided you give appropriate credit to the original author(s) and the source, provide a link to the Creative Commons license, and indicate if changes were made.

\section{References}

1. Davies DJ, Moran JE, Niall JF, Ryan GB (1982) Segmental necrotising glomerulonephritis with antineutrophil antibody: possible arbowirus aetiology? Br Med J (Clin Res Ed) 285(6342):606

2. van der Woude FJ, Rasmussen N, Lobatto S, Wiik A, Permin H, van Es LA, van der Giessen M, van der Hem GK, The TH (1985) Autoantibodies against neutrophils and monocytes: tool for diagnosis and marker of disease activity in Wegener's granulomatosis. Lancet 1(8426):425-429

3. Pendergraft WF 3rd, Nachman PH (2015) Recent pathogenetic advances in ANCA-associated vasculitis. Presse Med 44:223-229

4. Hutton HL, Holdsworth SR, Kitching AR (2017) ANCAassociated vasculitis: pathogenesis, models and preclinical testing. Semin Nephrol 37:418-435

5. Jarrot P-A, Kaplanski G (2016) Pathogenesis of ANCA-associated vasculitis: an update. Autoimmun Rev 15:704-713

6. Chen M, Kallenberg CGM (2010) The environment, geoepidemiology and ANCA-associated vasculitides. Autoimmun Rev 9:293-298

7. Popa ER, Stegeman CA, Kallenberg CGM, Cohen Tervaert JW (2002) Staphylococcus aureus and Wegener's granulomatosis. Arthritis Res 4:77-79

8. Konstantinov KN, Ulff-Moller CJ, Tzamaloukas AH (2015) Infections and antineutrophil cytoplasmic antibodies: triggering mechanisms. Autoimmun Rev 14:201-203

9. Popa ER, Cohen Tervaert JW (2003) The relation between Staphylococcus aureus and Wegener's granulomatosis: current knowledge and future directions. Intern Med 42:771-780

10. Pendergraft WF 3rd, Preston GA, Shah RR, Tropsha A, Carter CW Jr, Jennette JC, Falk RJ (2004) Autoimmunity is triggered by cPR-3 (105-201), a protein complementary to human autoantigen proteinase-3. Nat Med 10:72-79

11. Jennette JC, Wilkman AS, Falk RJ (1998) Diagnostic predictive value of ANCA serology. Kidney Int 53:796-798

12. Choi HK, Liu S, Merkel PA (2001) Diagnostic performance of antineutrophil cytplasmic antibody tests for idiopathic vasculitides: metaanalysis with a focus on antimyeloperoxidase antibodies. J Rheumatol 28:1584-1590

13. Vega LE, Espinoza LR (2016) Predictors of poor outcome in ANCA-associated vasculitis (AAV). Curr Rheumatol Rep 18:70 597. https://doi.org/10.1038/ki.2010.198

14. Rutgers A, Heeringa P, Damoiseaux JG, Tervaert JW (2003) ANCA and anti-GBM antibodies in diagnosis and follow-up of vasculitic disease. Eur J Intern Med 14:287-295

15. Fijolek J, Wiatr E, Petroniec V, Augustynowicz-Kopec E, Bednarek M, Gawryluk D, Martusewicz-Boros MM, Modrzewska K, Radzikowska E, Roszkowski-Sliz (2018) The presence of staphylococcal superantigens in nasal swabs and correlation with activity of granulomatosis with polyangiitis in own material. Clin Exp Rheumatol 36(Suppl 111):40-45

16. Jenette JC, Falk RJ, Bacon PA, Basu N, Cid MC, Ferrario F, FloresSuarez LF, Gross WL, Guillevin L, Hagen EC, Hoffman GS, Jayne DR, Kallenberg CG, Lamprecht P, Langford CA, Luqmani RA, Mahr AD, Matteson EL, Merkel PA, Ozen S, Pusey CD,
Rasmussen N, Rees AJ, Scott DG, Specks U, Stone JH, Takahashi K, Watts RA (2013) 2012 revised International Chapel Hill Consensus Conference nomenclature of vasculitides. Arthritis Rheum 65:1-11

17. Luqmani RA, Bacon PA, Moots RJ, Janssen BA, Pall A, Emery P, Savage C, Adu D (1994) Birmingham Vasculitis Activity Score (BVAS) in systemic necrotizing vasculitis. QJM 87:671-678

18. Fijolek J, Wiatr E, Gawryluk D, Martusewicz-Boros MM, Orlowski TM, Dziedzic D, Polubiec-Kownacka M, Oniszh M, Langfort R, Roszkowski-Sliz (2016) Intratracheal dilation-injection technique in the treatment of granulomatosis with polyangiitis patients with subglottic stenosis. J Rheumatol 43:2042-2048

19. Suwanochte S, Rachayon M, Rodsaward P, Wongpiyabovorn J, Deekajorndech T, Wright HL, Edwards SW, Beresford MW, Rerknimitr P, Chiewchengchol D (2018) Anti-neutrophil cytoplasmic antibodies and their clinical significance. Clin Rheumatol 37: 875-884

20. Aggarwal A, Sharma A, Rathi M, Sharma K, Minz RW (2015) Antineutrophil cytoplasmic antibodies (ANCA): role in disease pathogenesis, diagnosis, and monitoring ANCA associated vasculitis. Indian J Rheumatol 10:48-53

21. Boomsma MM, Stegeman CA, van der Leij MJ, Oost W, Hermans J, Kallenberg CG, Limburg PC, Tervaert JW (2000) Prediction of relapses in Wegener's granulomatosis by measurement of antineutrophil cytoplasmic antibody levels: a prospective study. Arthritis Rheum 43:2025-2033

22. Stegeman CA, Cohen Tervaert JW, Sluiter WJ, Manson WL, de Jong PE, Kallenberg CGM (1994) Association of chronic nasal carriage of Staphylococcus aureus and higher relapse rates in Wegener granulomatosis. Ann Intern Med 120:12-17

23. Han WK, Choi HK, Roth RM, McCluskey RT, Niles JL (2003) Serial ANCA titers: useful tool for prevention of relapses in ANCA-associated vasculitis. Kidney Int 63:1079-1085

24. Tomasson G, Grayson PC, Mahr AD, LaValley M, Merkel PA (2012) Value of ANCA measurements during remission to predict a relapse of ANCA-associated vasculitis - a meta-analysis. Rheumatology (Oxford) 51:100-109

25. Thai L-H, Charles P, Resche-Rigon M, Desseaux K, Guillevin L (2014) Are anti-proteinase-3 ANCA a useful marker of granulomatosis with polyangiitis (Wegener's) relapses? Results of a retrospective study on 126 patients. Autoimmun Rev 13:313-318

26. Girard T, Mahr A, Noel LH, Cordier JF, Lesavre P, Andre MH, Guillevin L (2001) Are antineutrophil cytplasmic antibodies a marker predictive of relapse in Wegener's granulomatosis? A prospective study. Rheumatology (Oxford) 40:147-151

27. Fussner LA, Hummel AM, Schroeder DR, Silva F, Cartin-Ceba R, Snyder MR, Hoffman GS, Kallenberg CG, Langford CA, Merkel PA, Monach PA, Seo P, Spiera RF, William St Clair E, Tchao BK, Stone JH, Specks U, Rituximab in ANCA-Associated VasculitisImmune Tolerance Network Research Group (2016) Factors determining the clinical utility of serial measurements of ANCA targeting proteinase 3. Arthritis Rheum 68:1700-1710

28. Sinico RA, Radice A, Corace C, Di Toma L, Sabadini E (2005) Value of a new automated fluorescence immunoassay for PR3 and MPO-ANCA in monitoring disease activity in ANCA-associated systemic vasculitis. Ann N Y Acad Sci 1050:185-192

29. Houben E, Bax WA, van Dam B, Slicker WA, Verhave G, Frerichs FC, van Eijk IC, Boerma WG, de Kuyper GT, Penne EL (2016) Diagnosing ANCA-associated vasculitis in ANCA positive patients: a retrospective analysis on the role of clinical symptoms and the ANCA titre. Medicine (Baltimore) 95(40):e5096

30. Finkielman JD, Merkel PA, Schroeder D, Hoffman GS, Spiera R, St Clair EW, Davis JC Jr, WJ MC, Lears AK, Ytterberg SR, Hummel AM, Viss MA, Peikert T, Stone JH, Specks U, WGET Research Group (2007) Antiproteinase 3 antineutrophil cytoplasmic 
antibodies and disease activity in Wegener granulomatosis. Ann Intern Med 147:611-619

31. Verstockt B, Bossuyt X, Vanderschueren S, Blockmans D (2015) There is no benefit in routinely monitoring ANCA titers in patients with granulomatosis with polyangiitis. Clin Exp Rheumatol 33(Suppl 89):72-76

32. Langford C (2012) Clinical features and dignosis of small-vessel vasculitis. Cleve Clin J Med 79(Suppl 3):3-7

33. Jones RB, Ferraro AJ, Chaudhry AN, Brogan P, Salama AD, Smith KG, Savage CO, Jayne DR (2009) A multicenter survey of rituximab therapy for refractory antineutrophil cytoplasmic antibodyassociated vasculitis. Arthritis Rheum 60:2156-2168

34. Khan A, Lawson CA, Quinn MA, Isdale AH, Green MJ (2010) Successful treatment of ANCA-negative Wegener's granulomatosis with rituximab. Int J Rheumatol 2010:846063

35. Heeringa P, Little MA (2011) In vivo approaches to investigate ANCA-associated vasculitis: lessons and limitations. Arthritis Res Ther 13:204

36. Stegeman CA, Cohen Tervaert JW, Sluiter WJ, Manson WL, de Jong PE, Kallenberg CG (1994) Association of chronic nasal carriage of Staphylococcus aureus and higher relapse rates in Wegener granulomatosis. Ann Intern Med 120:12-17

37. Xu SX, McCormick JK (2012) Staphylococcal superantigens in colonization and disease. Front Cell Infect Microbiol 2:1-11

38. Oliveira DBG (2018) Linked help from bacterial proteins drives autoantibody production in small vessel vasculitis. Med Hypotheses 112:24-26

39. Brinkmann V, Reichard U, Goosmann C, Fauler B, Uhlemann Y, Weiss DS, Weinrauch Y, Zychlinsky A (2004) Neutrophil extracellular traps kill bacteria. Sience 303:1532-1535

40. Draibe J, Rodo X, Fulladosa X, Martinez-Valenzuela L, DiazEncarnacion M, Santos L, Marco H, Quintana L, Rodriguez E, Barros X, Garcia R, Balius A, Cruzado JM, Torras J, Grupo de Malalties Glomerulas de la Societat Catalana de Nefrologia
(GLOMCAT) (2018) Seasonal variations in the onset of positive and negative renal ANCA-associated vasculitis in Spain. Clin Kidney J 11:468-473

41. Berti A, Dejaco C (2018) Update on the epidemiology, risk factors, and outcomes of systemic vasculitides. Best Pract Res Clin Rheumatol 32:271-294

42. Lionaki S, Blyth ER, Hogan SL, Hu Y, Senior BA, Jennette CE, Nachman P, Jennette JC, Falk RJ (2012) Classification of antineutrophil cytoplasmic autoantibody vasculitides. The role of antineutrophil cytoplasmic autoantibody specificity for myeloperoxidase or proteinase 3 in disease recognition and prognosis. Arthritis Rheum 64:3452-3462

43. Unizony S, Villarreal M, Miloslavsky EM, Lu N, Merkel PA, Spiera R, Seo P, Langford CA, Hoffman GS, Kallenberg CM, St Clair EW, Ikle D, Tchao NK, Ding L, Brunetta P, Choi HK, Monach PA, Fervenza F, Stone JH, Specks U, RAVE-ITN Research Group (2016) Clinical outcomes of treatment of anti-neutrophil cytoplasmic antibody (ANCA)-associated vasculitis based on ANCA type. Ann Rheum Dis 75:1166-1169

44. Bossuyt X, Rasmussen N, van Paassen P, Hellmich B, Baslund B, Vermeersch P, Blockmans D, Cohen Tervaert JW, Csernok E, Damoiseaux J (2017) A multicentre study to improve clinical interpretation of proteinase- 3 and myeloperoxidase anti-neutrophil cytoplasmic antibodies. Rheumatology 56:1533-1541

45. Glasner C, de Goffau MC, van Timmeren MM, Schulze ML, Jansen B, Tavakol M, van Wamel WJB, Stegeman CA, Kallenberg CGM, Arends JP, Rossen JW, Heeringa P, van Dijl JM (2017) Genetic loci of Staphylococcus aureus associated with anti-neutrophil cytoplasmic autoantibody (ANCA)-associated vasculitides. Sci Rep 7:12211

Publisher's note Springer Nature remains neutral with regard to jurisdictional claims in published maps and institutional affiliations. 